\title{
Acute Gastric Dilatation in Anorexia Nervosa
}

\author{
K. P. JENNINGS, A. M. KLIDJIAN
}

British Medical fournal, 1974, 2, 477-478

\section{Summary}

Two patients with anorexia nervosa were treated on a general surgical unit for acute gastric dilatation. In both cases the dilatation rapidly followed an increase in the usual low dietary intake of the patients, and the ingestion of extra food may have initiated the acute episode. Conservative treatment with parenteral fluids, nasogastric intubation, and then a gradual return to a normal diet proved a satisfactory method of management. In one patient the anorexia itself was improved.

\section{Introduction}

Two cases of acute gastric dilatation in patients undergoing treatment for anorexia nervosa have previously been reported. Russell (1966) described the case of a 16-year-old girl who developed acute dilatation two days after a high calorie fluid diet was begun. His patient responded to conservative management, but Russell thought there was a strong psychogenic element in the causation of the dilatation. Evans (1968) in a full review of acute dilatation of the stomach described a 20 -year-old girl with anorexia nervosa who had acute gastric dilatation five days after starting hospital dietary treatment. This patient's stomach rapidly dilated and perforated in two places, which naturally required surgical intervention. We describe two cases that conform to a similar pattern.

\section{Case 1}

A 14-year-old schoolgirl of Spanish extraction had developed anorexia nervosa over the 12 months before admission. Considering herself overweight she had started dieting and had reduced weight from $9 \mathrm{st}(57 \mathrm{~kg})$ to $6 \frac{1}{2} \mathrm{st}(41 \mathrm{~kg})$ in under six months. Her periods stopped during this time. She was unable to regain her appetite, however, and was urged by her family and by her general practitioner to seek hospital treatment. This she initially refused. In a short time she started to vomit everything she ate and continued to lose weight.

Two months later she eventually attended the medical outpatient department and was immediately admitted. Her main symptoms on admission were: complete loss of appetite, frequent vomiting, constipation, and amenorrhoea. She had no abdominal pain. On examination she was very thin, weighed 5 stones $(32 \mathrm{~kg}$ ), and looked dehydrated and pale. Her abdomen was tense but not greatly distended. A diagnosis of anorexia nervosa was firmly made and comfirmed on psyohiatric assessment.

In the ward she was coaxed into taking a light diet but, after 24 hours, this provoked worse vomiting, her abdomen was more distended, and she had severe abdominal pain. She became rapidly more dehydrated and parenteral fluids were started. On examination she now looked very ill with gross abdominal distension which was more marked in the upper abdomen. Her pulse rose to 150 beats/min and she became periphenally collapsed with extreme abdominal tenderness. A plain abdominal $x$-ray examination confirmed this to be a gastric dilation. A nasogastric tube was \footnotetext{
Surgical Department, St. Bartholomew's Hospital, London EC1A

K. P. JENNINGS, M.B., B.s. Senior House Officer

A. M. KLIDJIAN, M.B., B.s., Senior House Officer
}

passed and more than $3 \frac{1}{2}$ litres of fluid were immediately aspirated. She was closely observed over the next 24 hours.

An emergency barium meal examination showed no obvious pathologioal cause for the dilatation. After five days the nasogastric tube was removed but she started vomiting again so another was passed. This remained for a further eight days. All this time she was maintained on parenteral nutrition. After the 13th day a light diet was begun, which she managed to keep down.

She remained in hospital under psychiatric care for another 10 weeks, being treated with chlorpromazine, imipnamine, and a gradually increasing diet. On discharge she weighed 7 stones $(45 \mathrm{~kg})$. She maintained that weight and had no further attacks of acute gastric dilatation.

\section{Case 2}

A 25-year-old married secretary had been receiving psychiatric treatment for anorexia nervosa during the nine months before admission. She had been married for 12 months and claimed that she had started to lose weight then though she had had amenorrhoea for two years. She had recently been feeling better and had gone out for a meal with her husband. The day after she developed sudden abdominal pain, which became so severe over the next 48 hours that she sought hospital admission.

The pain was epigastric, unrelated to meals, and colicky in nature. She felt nauseated but had not been sick. She was not constipated. On examination she was a very thin, anxious, and pale girl who weighed just over 4 stones $(25 \mathrm{~kg})$. She was not clinically dehydrated. Her abdomen was distended and tender in the epigastrium. Bowel sounds were tympanitic, and a plain abdominal $x$-ray picture showed a huge gastric dilatation. The small bowel was also dilated with many fluid levels.

Parenteral fluids were started and a nasogastric tube passed. Three litres of fluid were aspirated, which resulted in a rapid improvement of the patient's condition. She remained on this regimen for 48 hours and was gently weaned on to a normal diet.

A barium meal examination was performed one month after she had overoome this acute episode and showed no residual dilatation of the stomach though the jejunal loops were still slightly dilated and the transit time through the bowel was slow. She had no further attacks of gastric dilatation though her anorexia proved more resistant to treatment, and she needed prolonged inpatient psychiatric care.

\section{Discussion}

Our first patient had been vomiting for about two months, but she did not develop her acute dilatation until she was admitted to hospital and put on a larger diet. The second girl developed her dilatation after an extra large meal two days before admission. Our cases together with those of Russell (1966) and Evans (1968) may give an indication of a possible aetiology of acute gastric dilatation.

Dragstedt et al. (1931) tried to determine the pathogenesis of acute dilatation in postoperative cases and concluded that an important factor was the building up of gastric, biliary, and intestinal secretions which, in turn, may result from nervous inhibition of normal gastric emptying. Markowski (1947) described acute dilatation in prisoners of war who were undernourished and subsequently fed rather rapidly. This sort of case closely resembles cases of first feeds of anorexic patients.

Thus the aetiology probably involves at least two factors which are interrelated. Firstly, patients have a strong emotional element and may overeact to being fed. They may be able to 
induce vomiting, which could then cause acute dilatation from hypokalaemia. Secondly, the hyperosmolar effect of introducing a relatively large amount of fluid and food into a stomach which has become used to less will cause dilatation.

Acute dilatation of the stomach is thus a recognized, if rare, complication of any patient suffering from anorexia nervosa who is beginning treatment. It seems that conservative management alone is adequate in the acute phase provided the condition is discovered before perforation of the stomach occurs. The mortality of uncomplicated gastric dilatation is low, but after perforation it is $80 \%$ (Evans, 1968).

We considered whether any medication the patients received before presentation might have caused the dilatation. One patient had been treated with diazepam and the other with chlorpromazine. We consulted Side Effects of Drugs (Meyler and Herxheimer, 1968) which made no reference to such effects after diazepam therapy. The phenothiazines have, however, on rare occasions been noted to cause a depression of gastric secretion leading to paralytic ileus in psychotic patients. This was noted in four out of 720 people studied. Since only one of our patients had received phenothiazines, however, we feel it is unlikely to have been a precipitating cause of presentation.

We thank Dr. N. C. Oswald and Mr. E. G. Tuckwell for permission to report these cases which were under their care and also Mr. W. M. Castleden for his comments and help.

\section{References}

Dragstedt, L. R., Montgomery, M. L., Ellis, J. C., and Matthews, W. R. (1931). Surgery, Gynecology and Obstetrics, 52, 1075.

Evans, D. S. (1968). British fournal of Surgery, 55, 940.

Markowski, B. (1947). British Medical fournal, $2,128$.

Meyler, L., and Herxheimer, A. (editors) (1968). Side Effects of Drugs, Vol. 6. Amsterdam, Excerpta Medica.

Russell, G. F. M. (1966). British Fournal of Psychiatry, 112, 203.

\section{Titanium Cranioplasty}

\section{S. GORDON, G. A. S. BLAIR}

British Medical fournal, 1974, 2, 478-481

\section{Summary}

The technique of repairing defects of the skull with titanium is described. The skull contour can be accurately reproduced. The technique is simpler than wiring or suturing methods. The material is inert, radiolucent, and rigid.

\section{Introduction}

One of the most conspicuous after effects of a depressed fracture of the skull or a penetrating missile injury is a disfiguring skull defect. Repair by cranioplasty is often needed. Frontal defects more than $2 \mathrm{~cm}$ in diameter are usually unsightly, and some cause discomfont, especially when the patient stoops. Larger defects leave the brain vulnerable to trauma, giving the patient a sense of insecurity.

A satisfactory cranioplasty should employ an onlay technique with a simple method of attaching the prosthesis to the skull. The material used should be inert in the tissues, radiolucent, easily and accurately shaped, capable of being adjusted at the time of insertion, and it should have sufficient mechanical strength to resist fracture or deformation under severe impact loading.

After the 1914-18 war autogenous bone was the material of choice for cranioplasty. But repair of a large defect requires a major operation to obtain the graft. Reproduction of the correct skull contour often proves difficult, and the patient often has more postoperative pain from the donor site than from the grafted area.

Some of the skull defects from the 1939-45 war were repaired with autopolymerized acrylic resin plates. At first they were preformed from a template or impression made at the finst operation. Spence (1954) described form-fioting plastic

\footnotetext{
Department of Neurosurgery, Royal Victoria Hospital, Belfast BT12 6BA

D. S. GORDON, M.CH., F.R.C.s., Consultant
}

Department of Dental Prosthetics, Dental School, Belfast BT12 6BA G. A. S. BLAIR, M.D.S., F.F.D., Senior Lecturer and Consultant cranioplasty in which the plastic was moulded into the defect during the hardening process. When the defeot is large, however, a smoothly contoured plate is difficult to shape and fit accurately.

Metals and alloys, including tantalum, titanium, stainless steel, and chrome-cobalt are not widely used because of the difficulty in forming the plate to the compound complex curvatures of the skull. Tantalum was used after the 1939-45 war (Woodhall and Spurling, 1947). It was shaped by a die/ counter die swaging technique or by hammering into a counter die (Lewin et al., 1948). Lewin and Gibson (1956) used tantalum to repair some of the skull defeots in British soldiers injured in Korea. The higher thermal conductivity of metals as compared with acrylic resin may limit their use for large plates in very hot climates (Spence, 1954.) For treating small skull defects plastic or metal plates or bone-grafting will usually prove satisfactory. The larger defect, however, poses special problems of contour and fixation which are difficult to solve by any of the available techniques.

In the civil disturbances in Northern Ireland over 900 people died between 1969 and 1973. In this type of urban guerilla warfare over $95 \%$ of penetrating injuries are gunshor wounds, many of them caused by high velocity bullets. The survivors usually have large skull defects, some with an area greater than $20 \mathrm{in}^{2}\left(129 \mathrm{~cm}^{2}\right)$.

We describe here how titanium has been used to repair these defects. The method combines accuracy with ease of insertion and is now used for skull defects of any size.

\section{Methods}

\section{INITIAL OPERATION}

Because of the risk of infection immediate cranioplasty is seldom advisable in patients with compound depressed skull fractures with brain laceration. The overlying skin is often severely lacerated and many wounds are heavily contaminated. Certain steps taken at the initial operation, however, will facilitate subsequent cranioplasty. A full-thickness skin cover for the defect and later for the prosthesis is essential. In missile wounds and depressed fractures caused by sharp objects the scalp laceration is often small and can be excised and sutured. A generous scalp flap is then cut about 2-3 cm beyond the limits of the skull fracture. This flap can be reflected 